\section{NUEVOS ESCENARIOS Y DESAFÍOS PARA LA CIENCIA ABIERTA. ENTRE EL OPTIMISMO Y LA INCERTIDUMBRE}

\author{
Mariano Fressoli \\ Conicet - CENIT/UNSAM \\ ORCID: 0000-0003-4060-0943 \\ mfressoli@unsam.edu.ar \\ Daniela De Filippo \\ Universidad Carlos III de Madrid \\ ORCID: 0000-0001-9297-9970 \\ dfilippo@bib.uc3m.es
}

\section{NEW SCENARIOS AND CHALLENGES FOR OPEN SCIENCE. BETWEEN OPTIMISM AND UNCERTAINTY}

Cómo citar este artículo/Citation: Fressoli, Mariano; De Filippo, Daniela (2021). Nuevos escenarios y desafíos para la ciencia abierta. Entre el optimismo y la incertidumbre. Arbor, 197(799): a586. doi.org/10.3989/arbor.2021.799001
Copyright: @ 2021 CSIC. Este es un artículo de acceso abierto distribuido bajo los términos de la licencia de uso y distribución Creative Commons Reconocimiento 4.0 Internacional (CC BY 4.0).
RESUMEN: En este artículo ofrecemos una visión sobre los nuevos escenarios que enfrenta la ciencia abierta en un contexto marcado por desafíos micro y macro estructurales que la pandemia de COVID-19 ha puesto de manifiesto. En primer lugar, se describen las políticas en el ámbito del acceso abierto, punta de lanza de la ciencia abierta y las resistencias que todavía encuentran estas prácticas. Se analiza, también, la participación de diferentes actores en los procesos de construcción de conocimiento científico, a través de prácticas de investigación participativa y ciencia ciudadana, así como en la creciente preeminencia de los datos. Se discuten, además, las principales tensiones estructurales que aparecen en los procesos de apertura analizando la construcción de políticas públicas sobre ciencia abierta. A modo de cierre, se introduce una reflexión sobre la situación actual en la que la epidemia del coronavirus está mostrando el rol cada vez más central que las prácticas abiertas tienen y tendrán en nuestra sociedad.

PALABRAS CLAVE: ciencia abierta, COVID-19, acceso abierto, ciencia ciudadana, datos abiertos.
ABSTRACT: This article provides an overview of the new scenarios facing Open Science in a context marked by the micro and macro structural challenges the COVID-19 pandemic has brought to light. First, policies are described in the field of open access, the launching pad for Open Science, and the resistance these practices still face. Also analysed is the participation of different social actors in processes to construct scientific knowledge, through participatory research and citizen science practices, as well as the growing supremacy of data. The main structural tensions that appear in open processes are also discussed, analysing the construction of public policies on Open Science. In closing, a reflection is presented on the current situation, where the coronavirus epidemic is showing the increasingly central role that open practices have and will have in our society.

KEYWORDS: Open Science, COVID-19, open access, citizen science, open data. 
La ciencia abierta se basa en prácticas de colaboración y apertura inspiradas, en gran medida, en el espíritu hacker y la construcción de experticias horizontales que desafían muchas de las convenciones establecidas de producción de conocimiento científico. El acceso abierto y las posibilidades de colaborar masivamente con actores heterogéneos permiten suponer una mayor eficiencia en la producción de conocimiento, transparencia en el uso de recursos y mayor adecuación entre las demandas sociales y la producción de conocimiento científico (Arza y Fressoli, 2017). Estos supuestos, reunidos en buena medida bajo el paraguas de la democratización del conocimiento, han generado un interés creciente por parte de científicos, hacedores de políticas y agencias y organismos de promoción y financiación de la investigación y desarrollo (I+D).

Al mismo tiempo, la transición hacia la ciencia abierta se produce en una época histórica convulsionada por nuevos desafíos globales como la epidemia del coronavirus, la crisis climática o las crecientes desigualdades sociales, económicas y de género que ponen en el centro de la escena el debate por el acceso y el control de la información y los datos, el creciente poder de las corporaciones y el papel de la ciudadanía.

Las características del escenario en el que se desarrolla actualmente la ciencia abierta, han servido de inspiración para el subtítulo de este monográfico -Entre el optimismo y la incertidumbre- en el que se presentan, discuten y analizan diferentes conceptos, elementos, recursos e iniciativas relacionados con las prácticas de ciencia abierta.

En esta presentación ofrecemos una visión sobre el impacto que la pandemia de la COVID-19 ha tenido en la aceleración y expansión de las prácticas de ciencia abierta. Se describen las políticas en el ámbito del acceso abierto, punta de lanza de la ciencia abierta. Se hace hincapié en la participación de diferentes actores en los procesos de construcción de conocimiento científico, a través de prácticas de investigación participativa y ciencia ciudadana, así como en la creciente preeminencia de los datos. Se mencionan las principales tensiones estructurales que aparecen en los procesos de apertura y se analiza la construcción de políticas públicas sobre ciencia abierta. A modo de cierre, reflexionamos sobre la situación actual en la que la epidemia del coronavirus está mostrando el rol cada vez más central que las prácticas abiertas tienen y tendrán en nuestra sociedad.

Los textos incluidos en este monográfico presentan una visión amplia y diversa sobre los desafíos que enfrenta actualmente la implementación de las prácticas de ciencia abierta en diferentes contextos. En esta línea hemos recogido aportaciones de diversos autores Iberoamericanos para conocer los alcances del movimiento en la región. Se incluyen, por un lado, artículos de investigación sobre el tema central y también se muestran otras tipologías documentales que, desde la sección de Materiales, aportan una mirada complementaria.

\section{LA CONSOLIDACIÓN DEL ACCESO ABIERTO.}

Las políticas de acceso abierto son la punta de lanza de las prácticas de ciencia abierta. Desde al menos finales de la década de 1990, diversos activistas y profesionales han abogado por la construcción de infraestructuras y prácticas que permitan el acceso abierto a publicaciones y, más recientemente, a datos. Muchas declaraciones pioneras de académicos y activistas que abocaban por el acceso abierto a la información científica, como las de Budapest (2002) ${ }^{1}$, Berlín (2003) ${ }^{2}$ y Bethesda $(2003)^{3}$, comenzaron en los últimos diez años a encontrar un fuerte eco institucional. Cada vez más organismos y programas de financiación científica, como la National Science Foundation en Estados Unidos y el Programa Horizonte 2020 de la Comisión Europea, han comenzado a promover decididamente políticas para compartir publicaciones en acceso abierto y/o a requerir la presentación de un plan de gestión de datos.

Siguiendo las recomendaciones de la Comisión Europea sobre el acceso a la información científica y su conservación (EUR-Lex, 2012), numerosos países han desarrollado políticas en esta línea: Finlandia con la Open Science and Research Iniciative (Finland, 2014; Forsström y Haataja, 2016), Holanda con el National Plan Open Science (Netherlands, 2017), Portugal con la adopción y aplicación de una política científica abierta (Portugal, 2016) o España, donde el Plan Estatal de Investigación Científica y Técnica y de Innovación 20172020 definió la apertura de resultados y datos de investigación obtenidos bajo financiación pública como

1 Véase https://www.budapestopenaccessinitiative.org/, visitado el 24 de noviembre de 2020.

2 Véase https://www.budapestopenaccessinitiative.org/, visitado el 24 de noviembre de 2020.

3 Véase http://legacy.earlham.edu/ peters/fos/bethesda.htm, visitado el 24 de noviembre de 2020. 
obligatoria (España, 2017). Fuera del ámbito europeo, también son notables los esfuerzos en gran número de países como Canadá o Sudáfrica (OECD, 2015) tal como han descrito Daniela De Filippo, Paulo Silva y María Manuel Borges (2019). En Latinoamérica, el movimiento de ciencia abierta también se hizo evidente de la mano de la apertura de las publicaciones científicas. Una de las iniciativas más destacadas es LA Referencia (Red Federada de Repositorios Institucionales de Publicaciones Científicas) creada en 2012, para apoyar las estrategias nacionales de acceso abierto en la región mediante una plataforma interoperable (LA Referencia, 2019). En esta región, ha sido también en la última década cuando han empezado a surgir políticas públicas para desarrollar y promover la ciencia abierta especialmente en países como Argentina, Brasil, Chile, Colombia, México, Perú y Uruguay, donde la promoción del desarrollo de infraestructuras para el acceso abierto ha dado lugar a la creación de repositorios nacionales (De Filippo y D’Onofrio, 2019).

Sin embargo, el avance de las políticas de acceso abierto todavía encuentra resistencias y obstáculos por parte de las universidades y de los mismos científicos.

Además, las iniciativas de acceso abierto se encuentran tensionadas por su relación con las editoriales comerciales. Esto ha dado lugar a propuestas como la del Plan $S$, con sus diez principios para alcanzar el acceso abierto en $2020^{4}$ o AMELICA, la infraestructura para la comunicación académica en abierto impulsada por UNESCO 5 .

Indudablemente el acceso abierto todavía constituye un campo de disputas y merece toda la atención por parte de los policy makers para consolidar sus avances.

Los ideales de colaboración y datos abiertos no siempre se traducen en prácticas concretas de apertura (Andreoli-Versbach y Mueller-Langer, 2014). Pocos artículos científicos presentan sus datos y mucho menos otras herramientas abiertas como el código de programación cuando se trata del desarrollo de software (Toelch y Ostwald, 2018). Tal como señalan Kaja Scheliga y Sascha Friesike (2014), la puesta en práctica de los valores de la ciencia abierta enfrenta una serie de obstáculos a nivel individual (miedo al robo de datos, falta de tiempo para realizar las tareas de puesta a punto y carga de los datos, falta de incenti- vos para publicar resultados negativos, etc.) así como barreras institucionales (falta de estándares claros para publicar en abierto, ausencia de infraestructura y/o soporte técnico, y falta de criterios de evaluación específicos). Claramente, a medida que las ideas de ciencia abierta se difunden y consolidan, se plantean desafíos a nivel de la aceptación de las nuevas prácticas, desarrollo de infraestructura adecuada y/o evaluación que pueden retrasar y/o impedir su avance.

Por ejemplo, muchas prácticas de apertura y colaboración online como los preprints se encuentran más difundidos en disciplinas como astronomía, matemáticas y físicas, pero son menos utilizados en ciencias sociales y humanas. Otras prácticas como la evaluación abierta de pares, se encuentra poco desarrollada entre las revistas de habla hispana y su implementación parece demorada más por barreras culturales que tecnológicas (Fressoli y Arza, 2018).

Esto plantea preguntas sobre cuáles son las mejores estrategias para promover las prácticas de apertura y de qué manera se pueden facilitar los aprendizajes entre diferentes comunidades de práctica. Sin dudas, una de las dificultades más reconocidas es el problema de la evaluación de las prácticas colaborativas. El sistema de evaluación tiende a favorecer la competencia y los logros individuales de la comunidad científica sobre la colaboración y el cuidado para compartir datos, publicaciones y otros elementos que podrían ser de utilidad para otros colegas y/o la sociedad. Cómo aprovechar las instancias de evaluación para favorecer la apertura y cuál (o cuáles) puede ser el locus institucional de este cambio es una de las preguntas más relevantes para el avance de la ciencia abierta en la próxima década. En el informe realizado para la Comisión Europea, O'Carroll y otros recomiendan cambios en forma de evaluación y financiamiento que incluyen el abandono de las métricas únicas por enfoques multidimensionales que se adecuen al puesto y las tareas demandadas (European Commission, 2017).

Un claro manifiesto de la necesidad de cambio en las prácticas y dinámicas científicas es la Declaración de Panamá en la que se hace evidente que «abrir la ciencia es ir más allá del acceso abierto" y se menciona la necesidad de transitar hacia modelos más colaborativos de creación, gestión, comunicación, preservación y apropiación entre el mundo académico, la sociedad y el Estado (Declaración de Panamá, 2018).

4 Véase https://www.openaire.eu/plan-s-a-european-open-access-mandate, visitado el 10 de noviembre de 2020.

5 Información disponible en: http://www.portal.amelica.org, visitado el 20 de noviembre de 2020. 


\section{DE LA INVESTIGACIÓN PARTICIPATIVA A LA CIEN- CIA CIUDADANA.}

Si bien, dentro del universo de la ciencia abierta, el acceso abierto sigue siendo la práctica más extendida, la ciencia ciudadana es uno de sus pilares fundamentales y se va consolidando como uno de los ámbitos con mayor potencial para promover la colaboración, la transparencia y la interacción entre diversos actores.

Los orígenes del movimiento pueden rastrearse en prácticas como la investigación-acción participativa que, desde sus comienzos en la década de los cuarenta del pasado siglo, ha tenido impacto en diversas disciplinas como educación, psicología o sociología. La intención de democratizar el conocimiento y hacer que las personas involucradas pasen de ser objeto de estudio a ser sujetos activos en las investigaciones, marcó un modo diferente de hacer ciencia que se ha visto potenciado con la irrupción del movimiento de ciencia abierta.

En cuanto al término ciencia ciudadana, sus orígenes pueden encontrarse en los postulados de Alan Irwin quien, en Reino Unido a mitad de los años noventa, la definía como "form of science developed and enacted by citizen themselves» (Irwin, 1995). Años más tarde Bonney hacía referencia a «public-participation engagement and science communication projects» (Riesch y Potter, 2014). Actualmente, al hablar de ciencia ciudadana se alude a un amplio espectro de actividades que van desde los proyectos diseñados por científicos en los que la ciudadanía participa a través de la recogida de datos (proyectos contributivos), otros en los que se ofrece a la sociedad oportunidades y herramientas de participación, tanto en el diseño del proyecto, como en la recogida de datos y en su posterior análisis (proyectos colaborativos) y, otros, en los que la ciudadanía participa en todas las fases (proyectos co-creativos).

Las iniciativas de ciencia ciudadana, lejos de ser acciones contracorriente, han ido obteniendo una atención política, institucional y pública cada vez más amplia. Un ejemplo de ello son los programas marco de la Unión Europea, en los que se han financiado varios proyectos de ciencia ciudadana como las acciones Foster que involucran a trece instituciones de ocho países, u otras como Socientize o Citi-sense, del VII Programa Marco. Incluso en el último programa Horizonte 2020 varias líneas de investigación se centran en esos temas y, en concreto la convocatoria Ciencia con y para la sociedad (SWAFS) es un ejemplo del interés en este nuevo enfoque.

Diversos ejemplos de ciencia abierta y participación ciudadana en disciplinas tan variadas como la salud (Belansky et al., 2011), el medio ambiente (Conrad y Hilchey, 2011; Sullivan et al., 2009) la astronomía (Raddick et al., 2013) o las ciencias sociales (Wright et al., 2017), han mostrado los beneficios de este tipo de práctica en las que el conocimiento científico va más allá del acceso abierto a la información y llega a diferentes actores sociales retroalimentando la producción de nuevo conocimiento.

Una de las iniciativas en que se hace más evidente la participación de la ciudadanía junto a los científicos son las llamadas science shops. Este tipo de entidades proporcionan apoyo independiente $\mathrm{y}$ participativo de investigación en respuesta a los problemas/necesidades de la sociedad civil. Nacidas a finales de los años setenta en Holanda, a partir de la iniciativa de un grupo de estudiantes para resolver problemas de su propia comunidad, la creación de estos centros ha supuesto un cambio muy importante en la dirección de la investigación. En los últimos años, su desarrollo ha estado muy ligado a la financiación de las grandes agencias internacionales. La Unión Europea mostró interés en este tipo de iniciativas a partir de la década del dos mil, al verlas como un punto de encuentro entre ciencia y sociedad. Esto dio origen a la financiación de diferentes proyectos como ISSNET, INTERACTS, SCIPAS, PERARES o Sci-shop.ue. y a la creación de la red Living Knowledge (The International Science Shop Network) a nivel europeo que se unió a la red Community-based Research Network en Estados Unidos y Canadá (De Filippo et al., 2018).

Sin dudas, la irrupción de la COVID-19 también ha tenido notable impacto en el ámbito de la ciencia ciudadana. A nivel europeo, una vez más, la Comisión Europea ha promovido iniciativas como el proyecto EU-Citizen.Science, una plataforma para compartir proyectos, recursos, herramientas y formación de ciencia ciudadana. Esta herramienta ofrece numerosas posibilidades de colaboración y permite llegar a actores muy diversos que se ven afectados por la pandemia: la ciudadanía que quiere ayudar a combatir el virus; la comunidad académica que requiere apoyo durante las interrupciones en sus trabajos de campo; los padres y madres que buscan recursos educativos para el hogar, etc. Asimismo, EUCitizen.Science permite conocer otras iniciativas como Flusurvey, un proyecto para supervisar tendencias de enfermedades 
infecciosas en la comunidad. La herramienta, creada durante la pandemia de gripe porcina de 2009, ha sido adaptada para supervisar enfermedades como la COVID-19 y ofrece información sobre la transmisión comunitaria, el riesgo de exposición, los cambios en el comportamiento de aquellos que requieren asistencia sanitaria y el cumplimiento de las recomendaciones para ayudar a limitar la propagación del coronavirus en la población general. Otra iniciativa similar, denominada Covid Near You, utiliza datos colectivos para visualizar mapas que ayuden a los ciudadanos y los organismos de salud públicos a identificar los actuales y potenciales puntos críticos de la COVID-19 en Canadá, los Estados Unidos y México.

Es indudable que el desarrollo de estas macro iniciativas no sería posible sin las grandes infraestructuras tecnológicas que permiten acceder, recoger, compartir y difundir diversos tipos de recursos. Sin embargo, aspectos como la recolección y el almacenamiento centralizados de la información también plantean un nuevo reto que puede empañar el potencial de la ciencia ciudadana. Es imposible analizar la ciencia ciudadana sin tener en cuenta los riesgos que afronta su inmensa capacidad de generación de datos e información y la necesidad de regular tanto el acceso a ellos como su uso y explotación. Es por ello que otro de los ámbitos que resulta interesante analizar es el de la ciencia de datos.

\section{LA CRECIENTE PREEMINENCIA DE LOS DATOS.}

Uno de los puntos más llamativos de la digitalización es el papel central que adquiere la ciencia de datos en la investigación científica. La ciencia de datos combina herramientas de estadística, minería de datos y aprendizaje automático para procesar grandes volúmenes de información. La disponibilidad de grandes cantidades de datos (data-driven) permite realizar hipótesis y teorías luego de su recolección (Hey, Tansley y Kole, 2009). La idea de ciencia impulsada por los datos requiere que los integrantes de la comunidad científica internacional compartan sus datos de forma abierta, reproducible e interoperable (Choudhury et al., 2014). El acceso abierto y la interoperabilidad aumenta la diversidad de grupos científicos, disciplinas e incluso sistemas inteligentes para leer $y$ analizar esos datos.

Autores como Michael Nielsen (2011) y Tony Hey, Steward Transley y Kristin Kole (2009) sugieren que la disponibilidad de grandes volúmenes de datos permitiría acelerar el análisis y la resolución de problemas y facilitar la democratización del conocimiento.
Estas afirmaciones no son extrañas a otras esferas de acción como los datos abiertos gubernamentales, y los datos abiertos para resolver problemas de desarrollo (ONU, 2014).

Al mismo tiempo, la preeminencia de los datos también parece impulsar una serie de cambios en las prácticas de producción e intercambio de conocimientos.

En primer lugar, el ascenso de las prácticas de data-driven science está comenzando a cambiar el perfil y las capacidades de los científicos. En un estudio reciente sobre ciencia digital realizado para el Banco Interamericano de Desarrollo (BID) (Arza et al., 2019) se encontró que el uso de herramientas de análisis estadístico, simulaciones, machine learning y otras técnicas, ya no son patrimonio de las ciencias sociales, biología y ecología sino que también comienzan a ser comunes en física, astronomía y/o ciencias de la tierra. Un indicador de estos cambios es el creciente uso de meta-análisis, integrando colecciones de estudios de diferentes investigadores. La creciente disponibilidad de revistas electrónicas, datos abiertos y nuevos procedimientos ha permitido acelerar el desarrollo de meta-análisis (Gurevitch et al., 2018).

Con la posibilidad de tomar más datos, la comunidad científica está aumentando la escala de su objeto de estudio, de las simples muestras a la posibilidad de estudiar poblaciones enteras o fenómenos a escala global. Esto mejora la calidad de la investigación y la eficiencia en el uso de recursos, ya que los mismos datos se convierten en insumo de distintas líneas o proyectos de investigación (Gurevitch et al., 2018).

Sin embargo, su uso no está ausente de riesgos. Los meta-análisis y otros usos de big data requieren muchas veces de infraestructura, procesamiento y capacidad de cómputo, recursos que no están disponibles para todos (Leonelli, Rappert y Davies, 2017). Estas disparidades pueden generar asimetrías entre diferentes instituciones y/o países. La necesidad de establecer procedimientos y herramientas que favorezcan el uso equitativo de los datos se ha vuelto una preocupación creciente entre científicos, activistas y organizaciones que promueven el uso de datos abiertos. Debido a que los datos abiertos de la ciencia también pueden ser utilizados por empresas y otros actores sociales, autores como Philip Mirowski (2018) advierten que la agenda de apertura implica rehacer las prácticas científicas de acuerdo a los lineamientos del capitalismo de plataformas. Tal como señalan Sabine Leonelli, Brian Rappert y Gail Davies (2017), los 
datos también pueden ser considerados un commodity que se adquiere en el mercado.

La comodificación de los datos no es para nada un fenómeno exclusivo de la ciencia abierta sino más bien señala una nueva fase de desarrollo del capitalismo caracterizado por la digitalización, la automatización y el uso intensivo de datos (Brynjolfsson y McAfee, 2014; Bastani, 2019). No obstante, estos procesos ponen de manifiesto el carácter crecientemente compartido (con empresas, organizaciones de la sociedad civil y organismos de gobierno) de muchas de las herramientas y procedimientos que utiliza la ciencia abierta.

Un aspecto complementario del uso intensivo de datos es la confluencia hacia herramientas abiertas comunes. Investigadores de disciplinas diversas como astronomía, ecología o biología se están volcando crecientemente al uso de software abierto como el programa $R$ o herramientas estadísticas desarrolladas en Python. Estas herramientas, al ser de código abierto, permiten desarrollar scripts y paquetes de análisis que se pueden compartir fácilmente en repositorios como Git-Hub o Source Forge ${ }^{6}$. El hecho de que expertos de disciplinas tan diferentes como economía y astronomía, biología molecular y sociología estén utilizando un mismo conjunto de herramientas indica sus propias oportunidades y desafíos. Por un lado, la colaboración en el uso de datos abiertos puede generar fricciones entre científicos y científicas de diferentes disciplinas (Edwards et al., 2011). Por otro lado, tal como la comodificación, el uso de herramientas abiertas amplía la colaboración hacia actores que disponen de diferentes formas de experticia no científica. Sin dudas, la participación de la ciudadanía no sólo es interesante para el diseño de políticas de investigación sino también para facilitar la divulgación de la ciencia e inclusive la colaboración con actores de fuera del ámbito académico. Pero, de la misma manera que la comodificación de los datos, el nuevo escenario de data-driven science obliga a preguntarse si es posible y en todo caso de qué manera los investigadores y las instituciones científicas van a establecer las fronteras entre aquello que es considerado dominio de la ciencia y aquello que no.

\section{TENSIONES ESTRUCTURALES DE LA APERTURA.}

Así, la ciencia abierta enfrenta de manera creciente tensiones estructurales en torno a la apertura del conocimiento. Por ejemplo, el escándalo por el uso ilegítimo de datos personales para marketing político en el cual la empresa Cambridge Analytica utilizó datos de Facebook para la campaña presidencial de EE.UU. de 2016, ha puesto en alerta a la población sobre la importancia de comprender y regular los usos ilegítimos de la información.

En la medida que la ciencia abierta promueve la publicación de los datos y otros insumos de investigación, no está exenta de estos riesgos. De forma similar, la apertura de los datos científicos puede favorecer la apropiación asimétrica por parte de instituciones que poseen mayores capacidades de procesamiento o mayores recursos, reforzando la posición subordinada de los sistemas científicos de los países o regiones en desarrollo. Por último, la creciente preocupación por la posverdad y la manipulación de la información por parte de diferentes actores llama la atención sobre la creciente crisis de legitimidad de los expertos reconocidos y del modelo informacional industrial que predominó en el siglo XX. La posverdad amenaza uno de los pilares fundamentales de la ciencia abierta, la capacidad de construir consensos para iniciar procesos de colaboración. Si bien la apertura de los datos parece favorecer el deseo de acceder a información de primera mano por parte del público, también expone a los científicos y científicas a nuevas presiones y requiere de nuevos compromisos y mecanismos institucionales para comunicar de forma accesible el conocimiento científico.

Estos fenómenos amenazan con subvertir los beneficios de la apertura y llaman la atención sobre la necesidad de desarrollar mecanismos para evitar la acción de intereses oportunistas, así como también los intentos de privatización de datos y otras formas de free riding.

De esta manera, en los últimos años, el imaginario optimista de la ciencia abierta parece enfrentarse tanto a desafíos micropolíticos asociados a la implementación de sus prácticas como a cambios macro-estructurales que ponen en cuestión la confianza en los

6 Las razones para adoptar estas herramientas son varias. Entre ellas destaca el ahorro de costes de licencias. Pero también están la posibilidad de acceder a una comunidad de soporte más activa que los softwares propietarios, la existencia de paquetes de análisis libremente disponibles y la facilidad para aprender online. El uso de software libre también permite a los investigadores re-utilizar scripts que fueron desarrollados para análisis muy específicos. En algunos casos, los investigadores pueden acompañar los datos con bitácoras de análisis, lo que facilita la reproducibilidad de la investigación. 
expertos y su papel en la construcción de políticas públicas.

\section{UN MOMENTO CRUCIAL: CIENCIA ABIERTA FREN- TE A LA COVID-19.}

La extraordinaria crisis del coronavirus ha generado una respuesta sin precedentes acelerando las prácticas de ciencia abierta y con ello la colaboración entre científicos a nivel global. La reacción del mundo científico se produjo, tanto a nivel de las prácticas de producción de conocimiento, como a nivel institucional y también a nivel ciudadano. Por un lado, los científicos y científicas de las disciplinas biomédicas comenzaron a utilizar rápidamente herramientas disponibles como repositorios abiertos de datos, publicaciones y preprints para acelerar la producción de conocimiento sobre el virus. Ejemplo de ello es el desciframiento y la puesta a disposición, en un repositorio abierto, de la secuencia genética del genoma del coronavirus sólo unas semanas después de anunciados los primeros casos en China ${ }^{7}$. Otro espacio donde la investigación abierta avanzó rápidamente alrededor de la pandemia ha sido el de las publicaciones. Desde enero 2020 la cantidad de artículos sobre el coronavirus no ha dejado de crecer. La semana del 20 de marzo el número de artículos publicados en formato preprint en bioRxiv llegaba a los 179 y otros 511 se publicaron en el repositorio abierto de ciencias médicas medRxiv. Para el 20 de agosto, la cantidad de artículos en preprint y evaluados por pares había ascendido a 80 mil (Besançon et al., 2020).

En enero de 2020, Wellcome Trust lanzó un llamado, firmado por 117 instituciones científicas, para asegurar que las instituciones médicas pudieran acceder a las publicaciones y datos relevantes para combatir la enfermedad. La declaración Sharing research data and findings relevant to the novel coronavirus (COVID-19) outbreak se basó en principios que ya habían sido enunciados durante las crisis recientes del virus del Zika y el Ébola en 2016. Poco tiempo después, en marzo de 2020, la Oficina de ciencia y tecnología de la Casa Blanca en Estados Unidos, junto a gestores y políticos de una docena de países, realizaron un segundo llamado para que las editoriales científicas de forma voluntaria permitieran el acceso abierto a los artículos relevantes sobre la COVID. De esta forma, la crisis de la COVID-19 ha reforzado el interés de dichas institu- ciones en la construcción de políticas y programas de ciencia abierta. En este contexto, uno de los procesos más notorios en el momento en el que se escribía esta presentación, era la intención de la UNESCO de realizar una recomendación sobre ciencia abierta a todos sus Estados miembros.

Los cambios que surgieron como respuesta a la COVID-19 superan ampliamente el ámbito de los resultados estrictamente científicos y se derraman sobre otros ámbitos sociales y técnicos. Una iniciativa llamativa es la Open Source Covid Pledge (liderada por un grupo internacional de investigadores, académicos y abogados) por la cual científicos, ingenieros y empresas se comprometen a liberar, utilizando licencias Creative commons, las patentes de diversos equipamientos técnicos (desde kits de diagnósticos, equipamiento médico, tratamientos, vacunas y sistemas de información) necesarios para atender la enfermedad (Contreras, Eisen y Peters, 2020). La iniciativa cubre más de 250 mil patentes y logró la firma de gigantes tecnológicos como Amazon, Facebook, Hewlett Packard Enterprise, IBM, Microsoft, entre otros ${ }^{8}$.

Por último, grupos de activistas de datos, hackers y makers también realizaron diversas contribuciones cercanas a los preceptos de ciencia abierta. Sirvan de ejemplo Frena la curva, una plataforma de innovación ciudadana que fue replicada en veintidós países (en su mayoría de habla hispana) que promueve formas de innovación social y cooperación e intercambio de recursos en base a herramientas de código abierto, y Crowdfight Covid, una plataforma que reúne más de cuarenta y cinco mil científicos, investigadores y personal de salud que comparten recursos y conocimientos para combatir el coronavirus. Las formas de cooperación amplia entre científicos y ciudadanos que tienen una larga tradición en ciencia abierta y ciudadana no sólo se adaptaron a la recolección de información sobre la pandemia, como en el caso de FOLDIT (uno de los proyectos de investigación pioneros en este campo), sino que también se extendieron hacia otros ámbitos como la divulgación de información científica fiable y el chequeo de desinformación.

Una revisión completa de las diferentes iniciativas de ciencia abierta que surgieron como respuesta a la pandemia excede con creces las intenciones de esta presentación. Es probable que la historia y la sociología de la ciencia, entre otras disciplinas, se demoren

7 Consultar post en: https://virological.org/t/novel-2019-coronavirus-genome/319, visitado el 20 de noviembre de 2020.

8 Para mayor información consultar: https://creativecommons.org/2020/04/20/tech-giants-jointhe-cc-supported-open-covid-pledge/ 
años en el estudio de lo que sucedió durante el transcurso de la pandemia. En lugar de intentar esta tarea imposible, en lo que sigue exploramos cuatro temáticas (acceso abierto, ciencia ciudadana, datos abiertos y las tensiones estructurales de la apertura) cuyo desarrollo era posible vislumbrar antes de 2020 y que ahora adquieren más relevancia para pensar el futuro de la ciencia abierta. En este contexto, el objetivo de este monográfico es explorar, en sentido amplio, las oportunidades y los desafíos diversos que enfrentan las prácticas de ciencia abierta. Se busca así comprender los diferentes problemas y fortalecer el debate sobre iniciativas, políticas y conceptos.

\section{CONTRIBUCIONES DE ESTE MONOGRÁFICO.}

Para profundizar en los aspectos mencionados, se presentan a continuación seis contribuciones, cuyos autores, todos ellos especialistas en el tema de la ciencia abierta, desarrollan sus investigaciones principalmente en el campo de la Documentación y de las Ciencias de la Información. Contar con representantes de España, Portugal, Brasil y Argentina pertenecientes a diversos entornos institucionales, permite dar un panorama amplio de las percepciones sobre el desarrollo de la ciencia abierta en Iberoamérica.

En primer lugar, el ensayo de Eva Méndez permite conocer la situación actual que vive Europa en términos de formulación e implementación de políticas sobre ciencia abierta. La autora ofrece en este trabajo un completo análisis desde su visión basada en la experiencia como chair en la European Open Science Platform (2018-2020). El texto revisa el concepto de open science en su interacción con open knowledge (conocimiento abierto) y analiza los principales retos de la ciencia abierta. Asimismo, ofrece una reflexión sobre la puesta en práctica de la open science, desde dos aproximaciones: la creación de políticas y estrategias formales a nivel de Estados miembros, y la implementación a través de Compromisos Prácticos de Implementación (PCls) de los agentes implicados.

El texto de Ernest Abadal sobre componentes de la ciencia abierta, ofrece una revisión general del tema analizando los antecedentes del concepto y sus diferentes definiciones. Destaca entre las principales características de este movimiento la apertura, la transparencia y la reutilización de contenidos. El artículo trata la ciencia abierta como un rompecabezas en el que diversas piezas como el acceso abierto, los datos de investigación, la revisión en abierto, los preprint, la ciencia ciudadana y los nuevos modelos de evaluación muestran un nivel de desarrollo desigual por lo que, según el autor, el principal desafío para una plena implementación es lograr un encaje adecuado de todos los componentes.

Por su parte Sarita Albagli y Luana Rocha presentan los resultados de una investigación en la que se analiza el papel de la ciencia ciudadana para hacer frente a los problemas que plantea la pandemia de la COVID-19. El estudio, centrado en el caso de Brasil, pone de manifiesto la relevancia de las acciones participativas para la reducción de riesgos de desastres como el de la pandemia. Esta investigación pone en evidencia el papel central que tiene la producción ciudadana de datos, información y conocimientos relevantes para la prevención, resiliencia y gestión de riesgos; y, por otro, amplía el alcance de la ciencia ciudadana para hacer frente a situaciones de emergencia y catástrofe.

Los aspectos económicos relacionados con el coste de la apertura del conocimiento y, en concreto con los cargos de publicación derivados del acceso abierto, son discutidos en el trabajo de Antonia Ferrer, Rafael Aleixandre, Cristian Vidal y Juan Carlos Valderrama. El artículo analiza la dualidad generada por la necesidad/ obligación de publicar en abierto los resultados de la investigación financiada con fondos públicos y los costes que esto genera para las agencias o instituciones financiadoras. Para ello se presenta un estudio de caso de publicaciones de autoría española en cinco disciplinas de ciencias sociales y humanidades. A través del uso de metodologías bibliométricas se han identificado las revistas de publicación, el coste de los cargos por procesamiento de artículos (APC) y los modelos más frecuentes de publicación en abierto. El trabajo, además de identificar los patrones de difusión de unas disciplinas concretas, permite ahondar en la discusión sobre el coste que deben afrontar las instituciones a la hora de promover y financiar la publicación en abierto, un aspecto central a tener en cuenta al gestionar e implementar políticas y estrategias de ciencia abierta.

Uno de los aspectos más discutidos en la implementación de iniciativas de ciencia abierta continúa siendo la evaluación de la actividad científica y tecnológica, dado que su concreción parece no lograr un consenso adecuado entre los diferentes actores involucrados. Es por ello que conocer la experiencia de evaluación desarrollada en Portugal -y presentada en el artículo de Ligia Ribeiro, María Manuel Borges y Diana Silva- puede señalar las ventajas y limitaciones de este tipo de práctica. El texto comenta la experiencia del Grupo de Trabajo sobre evaluación de la ciencia (grupo de trabalho sobre avaliação da 
ciência), puesto en marcha en 2016, haciendo especial hincapié en las recomendaciones del subgrupo de Evaluación Científica. Según mencionan las autoras, estas recomendaciones, sumadas a las de los restantes grupos de trabajo, han sido fundamentales para la implementación de las prácticas de Ciencia abierta en diferentes ámbitos.

Entre las buenas prácticas relacionadas con la producción y difusión del conocimiento, la transparencia, la reproductibilidad y el acceso a datos y resultados, se han convertido en elementos centrales del proceso de investigación. En este tema profundiza el artículo de José Luis Ortega quien analiza el naciente movimiento OpenCitations explorando las posibilidades y ventajas que brinda tanto para la difusión como para la evaluación de la actividad investigadora. El texto realiza una revisión de los orígenes y evolución de este movimiento que busca hacer de las citas bibliográficas un bien común para la comunidad científica. Se menciona además, el cambio que se está generando en el mercado de la documentación científica con al surgimiento de nuevos productos y plataformas, y la oportunidad para el desarrollo de portales regionales o institucionales que, alimentados de estas fuentes abiertas, permitan un evaluación propia e independiente.

Por último, el monográfico se complementa con dos documentos enlazados en la sección de Materiales que amplían la mirada para conocer la situación actual que vive la ciencia abierta. Dos textos, uno de Leslie Chan y otro de Carolina Botero, que presentan e introducen el Manifiesto de Ciencia abierta y colaborativa, y la Declaración de Panamá respectivamente.

\section{REFERENCIAS}

Andreoli-Versbach, Patrick y Mueller-Langer, Frank (2014). Open access to data: An ideal professed but not practised. Research Policy, 43(9): 1621-1633. doi:10.1016/j.respol.2014.04.008

Arza, Valeria y Fressoli, Mariano (2017). Systematizing benefits of open science practices. Information Services and Use, 37(4): 463-474. Disponible en: https://content.iospress.com/articles/ information-services-and-use/isu861. doi: 10.3233/ISU-170861

Arza, Valeria; Fressoli, Mariano; Actis, Guillermina y del Castillo, Martín (2019). La ciencia abierta en América Latina. Reporte realizado para el Banco Interamericano de Desarrollo. Montevideo, Mimeo.

Bastani, Aaron (2019). Fully Automated Luxury Communism: a manifiesto, London: Verso Books.

Belansky, Elaine; Cutforth, Nick; Chavez, Robert; Waters, Emily y BartlettHorch, Kandiss (2011). An adapted version of intervention mapping (AIM) is a tool for conducting community-based participatory research. Health Promot. Pract, 12: 440-455.

Besançon, Lionni, Peiffer-Smadja, Nathan, Segalas, Corentin, Jiang, Haiting, Masuzzo, Paola, Smout, Cooper, Deforet, Maxime y Leyrat, Clémence (2020), Open Science Saves Lives: Lessons from the COVID-19 Pandemic, BioRxiv. doi: 10.1101/2020.08.13.249847
Berlin Declaration on Open Access to Knowledge in the Sciences and $\mathrm{Hu}$ manities, (2003). Accesible en: https:// openaccess.mpg.de/Berlin-Declaration

Bethesda Statement on Open Access Publishing, (2003). Accesible en: http:// legacy.earlham.edu/ peters/fos/bethesda.htm

Brynjolfsson, Erick y McAfee, Andrew (2014). The second machine age: Work, progress, and prosperity in a time of brilliant technologies. New York: W. W. Norton \& Company.

Choudhury, Suparna; Fishman, Jennifer R.; McGowan, Michelle L. y Juengst, Eric T. (2014). Big data, open science and the brain: lessons learned from genomics. Frontiers in Human Neuroscience, 8: 239. doi: 10.3389/fnhum.2014.00239

Conrad, Cathy y Hilchey, Krista (2011). A review of citizen science and community-based environmental monitoring: Issues and opportunities. Environ. Monit. Assess, 176: 273-291.

Contreras, Jorge; Eisen, Michel y Peters Diane (2020). COVID-19: save lives with open intellectual-property licences. Nature 583, 683. doi:10.1038/d41586020-02233-4

Declaración de Panamá para la Ciencia Abierta (2018). Recuperado de https://hiperderecho.org/wp-content/ uploads/2018/11/declaracion_panama_ciencia_abierta.pdf

De Filippo, Daniela; Silva, Paulo; Borges, María Manuel (2019). Caracterización de las publicaciones de España y Portugal sobre Open Science y análisis de su presencia en las redes sociales. Revista Española de Documentación Científica, 42 (2), e235. doi:.10.3989/ redc.2019.2.1580

De Filippo, Daniela y D’Onofrio, María Guillermina (2019). Alcances y limitaciones de la ciencia abierta en Latinoamérica: análisis de las políticas públicas y publicaciones científicas de la región. $\mathrm{Hi}$ pertext.net, (19): 32-48. doi:10.31009/ hipertext.net.2019.i19.03

De Filippo, Daniela; Bautista-Puig, Nuria; Mauleón, Elaba; Sanz-Casado, Elías (2018). A Bridge between Society and Universities: a Documentary analysis of science shops. Publications 6 (3): 36. doi:10.3390/publications6030036

Edwards, Paul; Mayernik, Matew; Batcheller, Archer; Bowker, Geofrey y Borgman, Cristine (2011). Science friction: Data, metadata, and collaboration. Social Studies of Science, 41(5): 667-690. Accesible en: http://www.jstor.org/stable/41301955

España. Ministerio de Economía, Industria y Competitividad, Secretaría de Investigación, Desarrollo e Innovación (2017). Plan Estatal de Investigación Científica y Técnica y de Innovación 2017-2020. Accesible en: http://www.idi.mineco. gob.es/stfls/MICINN/Prensa/FICHEROS/2018/PlanEstatalIDI.pdf

EUR-Lex (2012). COMMISSION RECOMMENDATION of 17 July 2012 on access to and preservation of scientific information 
(2012/417/EU). Official Journal of the European Union, 39-43. Accesible en: https://publications.europa.eu/en/publication-detail/-/publication/48558fc9-d4c 8-11e1-905c-01aa75ed71a1

European Commission (2017) Evaluation of Research Careers fully acknowledging Open Science Practices. Accesible en: https://ec.europa.eu/research/openscience/pdf/os_rewards_wgreport_final. pdf

Finland. The Ministry of Education and Culture's Open Science and Research Initiative 2014-2017 (2014). The Open Science and Research Roadmap. Reports of the Ministry of Education and Culture, Finland, 2014:21. Accesible en: http:// julkaisut.valtioneuvosto.fi/bitstream/ handle/10024/75210/okm21.pdf

Forsström, Pirjo-Leena; Haataja, Juha. (2016). Open Science as an Instrument for Effective Research. Signum, 2 : 11-15. Accesible en: http://ojs.tsv.fi/index.php/ signum/article/viewFile/58741/20303

Fressoli, Mariano y Arza, Valeria (2018). Los desafíos que enfrentan las prácticas de ciencia abierta. Teknokultura. Revista de cultura digital y movimientos sociales, 15(2): 429-448. doi: 10.5209/ TEKN.60616

Gurevitch, Jessica; Koricheva, Julia; Nakagawa, Shinichi y Steward, Gavin (2018). Meta-analysis and the science of research synthesis. Nature 555: 175182. doi: $10.1038 /$ nature 25753

Hey, Tony; Tansley, Steward y Tolle, Kristin (2009). The Fourth Paradigm: Data-Intensive Scientific Discovery. Redmond, WA: Microsoft research.
Irwin, Alan (1995). Citizen Science: a Study of People, Expertise and Sustainable Development. Routledge: Abingdon, UK.

LA Referencia: Red de Repositorios de Acceso Abierto a la Ciencia (2019). Compartiendo y dando visibilidad a la producción científica en América Latina. Accesible en: http://www.lareferencia.info/es/institucional/quienes-somos

Leonelli, Sabine; Rappert, Brian y Davies, Gail (2017). Data Shadows: Knowledge, Openness, and Absence. Science, Technology, \& Human Values, 42(2): 191202. doi:10.1177/0162243916687039

Mirowski, Philip (2018). The future(s) of open science. Social Studies of Science, 48(2): 171-203. doi:10.1177/0306312718772086

Netherlands. Dutch Ministry of Education, Culture and Science (2017). National Plan Open Science. doi: 10.4233/uuid:9e9fa82e-06c1-4d0d-9e20-5620259a6c65

Nielsen, Michael (2011). Reinventing discovery: the new era of networked science. Princeton University Press.

OECD (2015). Making Open Science a Reality. OECD Science, Tecnology and Industy Policy Papers, 25. Paris: OECD Publications. doi: $10.1787 / 5$ jrs2f963zs1-en

ONU (2014). A world that counts. Mobilising the data revolution for sustainable development. Accesible en: https://www. undatarevolution.org/wpcontent/uploads/2014/11/A-World-That-Counts.pdf

Portugal. Ministério da Ciência, Tecnologia e Ensino Superior (MCTES) (2016). Ciência Aberta, Conhecimento para todos: Princí- pios orientadores. Accesible en: https:// www.fosteropenscience.eu/content/ ciencia-aberta-conhecimento-para-todos

Raddick, Jordan; Bracey, Georgia; Gay, Pamela; Lintott, Chris; Cardamone, Carie; Murray, Phil; Schawinski, Kevin; Szalay, Alexander y Vandenberg, Jan (2013). Galaxy Zoo: Motivations of citizen scientists. arXiv preprint arXiv:1303.6886.

Riesch, Hauke y Potter, Clive (2014). Citizen Science as seen by scientists: Methodological, epistemological and ethical dimensions. Public Understanding of Science, 23: 107-120.

Scheliga, Kaja y Friesike, Sascha. (2014). Putting open science into practice: A social dilemma? First Monday, 19(9). doi: 10.5210/fm.v19i9.5381

Sullivan, Braian; Wood, Chris; lliff, Marshal; Bonney, Robert; Fink, Daniel y Kelling, Steve. (2009) eBird: A citizen-based bird observation network in the biological sciences. Biological Conservation, 142: 2282-2292.

Toelch, Ulf y Ostwald, Dirk (2018). Digital open science-Teaching digital tools for reproducible and transparent research. PLoS Biology, 16(7). doi: 10.1371/journal. pbio.2006022

Wright, Leslie; King, Diane; Retrum, Jessica; Helander, Kenneth; Wilkins, Shari; Boggs, Jennifer; Portz, Jennifer; Nearing, Kathryn y Gozansky, Wendolyn (2017). Lessons learned from community-based participatory research: Establishing a partnership to support lesbian, gay, bisexual and transgender ageing in place. Family Practice, 34(3): 330-335. doi: 10.1093/fampra/cmx005 